\title{
Linking an Anxiety-Related Personality Trait to Cardiac Autonomic Regulation in Well-Defined Healthy Adults: Harm Avoidance and Resting Heart Rate Variability
}

\author{
Lien-Cheng Kao', Yu-Wen Liư², Nian-Sheng Tzeng ${ }^{2,3}$, Terry B. J. Kuo ${ }^{4}$, \\ San-Yuan Huang ${ }^{2}$, Chuan-Chia Chang ${ }^{2}$, and Hsin-An Chang ${ }^{2} \bowtie$ \\ ${ }^{1}$ Department of Psychiatry, Beitou Branch, Tri-Service General Hospital, National Defense Medical Center, Taipei, Taiwan \\ ${ }^{2}$ Department of Psychiatry, Tri-Service General Hospital, National Defense Medical Center, Taipei, Taiwan \\ ${ }^{3}$ Student Counseling Center, National Defense Medical Center, Taipei, Taiwan \\ ${ }^{4}$ Institute of Brain Science, National Yang-Ming University, Taipei, Taiwan
}

\begin{abstract}
Objective Anxiety trait, anxiety and depression states have all been reported to increase risks for cardiovascular disease (CVD), possibly through altering cardiac autonomic regulation. Our aim was to investigate whether the relationship between harm avoidance (HA, an anxiety-related personality trait) and cardiac autonomic regulation is independent of anxiety and depression states in healthy adults.

Methods We recruited 535 physically and mentally healthy volunteers. Participants completed the Beck Anxiety Inventory (BAI), Beck Depression Inventory (BDI) and Tri-dimensional Personality Questionnaire. Participants were divided into high or low HA groups as discriminated by the quartile value. Cardiac autonomic function was evaluated by measuring heart rate variability (HRV). We obtained the time and frequency-domain indices of HRV including variance (total HRV), the low-frequency power (LF; $0.05-0.15 \mathrm{~Hz}$ ), which may reflect baroreflex function, the high-frequency power (HF; $0.15-0.40 \mathrm{~Hz}$ ), which reflects cardiac parasympathetic activity, as well as the LF/ HF ratio.

Results The BDI and HA scores showed associations with HRV parameters. After adjustment for the BDI scores and other control variables, HA is still associated with reduced variance, LF and HF power. Compared with the participants with low HA, those with high HA displayed significant reductions in variance, LF and HF power and a significant increase in their LF/HF ratio.

Conclusion This study highlights the independent role of HA in contributing to decreased autonomic cardiac regulation in healthy adults and provides a potential underlying mechanism for anxiety trait to confer increased risk for CVD.
\end{abstract}

Psychiatry Investig 2016;13(4):397-405

Key Words Heart rate variability, Anxiety trait, Harm avoidance, Depression, Anxiety.

\section{INTRODUCTION}

Depression $^{1}$ and anxiety ${ }^{2}$ have been associated with in-

Received: July 18, 2015 Revised: September 19, 2015

Accepted: October 24, 2015 Available online: March 23, 2016

$\triangle$ Correspondence: Hsin-An Chang, MD

Department of Psychiatry, Tri-Service General Hospital, National Defense Medical Center, No. 325, Cheng-Kung Road, Sec. 2, Nei-Hu District, Taipei 114, Taiwan Tel: +011-886-2-8792-7220, Fax: +011-886-2-8792-7221

E-mail: chang.ha@mail.ndmctsgh.edu.tw

$\triangle$ Correspondence: Chuan-Chia Chang, MD

Department of Psychiatry, Tri-Service General Hospital, National Defense Medical Center, No. 325, Cheng-Kung Road, Sec. 2, Nei-Hu District, Taipei 114, Taiwan Tel: +011-886-2-8792-7220, Fax: +011-886-2-8792-7221

E-mail: chang.ha@mail.ndmctsgh.edu.tw

(a) This is an Open Access article distributed under the terms of the Creative Commons Attribution Non-Commercial License (http://creativecommons.org/licenses/by$\mathrm{nc} / 3.0$ ) which permits unrestricted non-commercial use, distribution, and reproduction in any medium, provided the original work is properly cited. creased risks of cardiovascular disease (CVD). Cardiac autonomic dysregulation is considered to be an important pathophysiologic factor and has been studied extensively in patients with clinical depression or anxiety. ${ }^{2,3}$ The smaller body of research conducted on healthy individuals have mixed results. ${ }^{4-6}$ Recent research on healthy subjects has leaned towards focusing on the relationship between personality traits and cardiac autonomic regulation.

Anxiety trait as a personality dimension is thought to represent an important predisposing factor for depression and anxiety-related disorders. It has also been reported to increase the risk for $\mathrm{CVD}^{7-9}$ though the definite mechanisms involved are not clear. Among the anxiety trait spectrum, harm avoidance (HA) is a well-known anxiety-related personality trait. ${ }^{10}$ Individuals with high-HA are fearful, pessimistic, socially in- 
hibited, easily tired, and prone to experience depression and anxiety disorders. ${ }^{11}$ Not only is HA associated with mental disorders, but it is measurable in non-affected subjects. Thus, this personality trait may represent a useful endophenotype to study the biological correlation of the vulnerability factors in the general population. Recent studies sought to examine the relationship between HA and cardiac autonomic regulation. The results are promising and seem to provide potential linkage between this personality trait and increased risk for CVD. ${ }^{4,12,13}$

However, research in this area has been hampered by the enrollment of smokers and the lack of an objective procedure and a structural clinical interview to exclude possible physical and psychiatric confounds, respectively. Thus, the reported associations should be validated by large-scale studies using suited sample to minimize the effects of potential confounding factors on cardiac autonomic regulation.

Heart rate variability refers to the complex beat-to-beat variation in heart rate produced by the interplay of sympathetic and parasympathetic (vagal) neural activity at the sinus node of the heart. Increased HRV reflects a healthy autonomic nervous system that is able to respond to environmental demands. ${ }^{14}$ Contrarily, low HRV is an indicator of autonomic inflexibility ${ }^{15}$ and a predictor of poor health status. ${ }^{16}$ Shortterm power spectral analysis of HRV, standardized since $1996,{ }^{17}$ is a reliable and non-invasive tool to probe the autonomic regulation of the heart. Distinct frequency bands of the heart rate signal are separated through this analysis, and their quantified relative intensity made through this analysis is called power. Low frequency $(\mathrm{LF})$ range power $(0.04-0.15 \mathrm{~Hz})$ is mediated by both sympathetic and parasympathetic activity, while high frequency range power $(\mathrm{HF})(0.15-0.4 \mathrm{~Hz})$ is mediated primarily by parasympathetic activity. The ratio of LF to HF (LF/ $\mathrm{HF}$ ) can be used to assess the comparative balance of the two branches of the autonomic nervous system on cardiac activity.

The study design is based on the following hypotheses: 1) there is a meaningful correlation between the personality trait HA and autonomic functioning as indexed by HRV; 2) in healthy subjects, the influence of HA on HRV is independent of the influence of psychological states (i.e., subclinical depression and/or anxiety) and other confounding factors; and 3) the subjects with high HA show lower HRV as compared to those with low HA.

\section{METHODS}

\section{Participants}

This study was approved by the Institutional Review Board for the Protection of Human Subjects of the Tri-Service General Hospital, a medical teaching hospital of the National De- fence Medical Centre in Taipei, Taiwan. All of participants were aged from 20 to 65 years, and all provided written informed consent. Participants came from the Taiwan Study of Depression and Anxiety (TAISDA), an ongoing cohort study conducted to examine the effect of depression and anxiety disorders on HRV as described elsewhere. ${ }^{18-20}$ In brief, after detailed questionnaire screening, chart review, clinical examination, electrocardiography, and relevant laboratory investigations, the subjects who were pregnant, had cancer, postural hypotension, vasovagal syncope, cardiovascular, respiratory, neurological, or endocrinological disorders that affect HRV, or those engaged in regular and strenuous physical training were excluded. Current or past smokers were also excluded. All participants were drug-naïve or had not used psychotropic medications or any medications that have been reported to affect autonomic functioning (e.g., antipsychotics, anticholinergics, antidepressants, oral contraceptives, anticonvulsants, anxiolytics, cerebral metabolic activators, or cerebral vasodilators) for at least 1 month prior to the beginning of the study. Each participant was evaluated using the Chinese version of the Modified Schedule of Affective Disorder and SchizophreniaLifetime (SADSL) $)^{21}$ to exclude those with psychiatric conditions. A total of 535 participants with valid data on both clinical ratings and HRV were included in the final statistical analysis.

\section{Control variables}

Previous findings have revealed that gender, age, body mass index (BMI), physical activity, and alcohol use are among the factors significantly affecting the autonomic control of heart rate. ${ }^{22-26}$ These factors were thus selected as control variables. Subjects reported the average frequency of physical exercise per week (A) and hours per session spent in purposeful exercise (B) in the past 6 months. "A" was rated with a five-point scale according to the frequency of exercise involving heavy breathing and sweating as "never", "seldom", "once a week", "twice a week", and "more than twice a week" ${ }^{27}$ The participants' self-reported weekly habitual physical activity was calculated by the formula: $\mathrm{A} \times \mathrm{B}$. Alcohol use, assessed with two items of the Alcohol Use Disorder Identification Test questionnaire, ${ }^{28}$ was defined by the average frequency of drinking and the number of drinks consumed on a typical drinking day in the past year. From these items, we derived the average amount of alcoholic drinks per day, with one drink defined as a standard drink, i.e., having the equivalent of 10 grams of alcohol. ${ }^{29}$

\section{Assessment of anxiety/depression severity}

All participants were assessed using the self-reported measures of anxiety and depression, i.e. Beck Anxiety Inventory 
(BAI) and Beck Depression Inventory (BDI).

\section{Personality assessment}

Personality traits were measured by the Chinese version of the Tri-dimensional Personality Questionnaire (TPQ), a 100item, self-administered, True-False instrument. Cronbach's a of novelty seeking (NS) was 0.70 while that of harm avoidance (HA) was $0.87 .{ }^{30}$ We also explored the NS dimension in the present study because this personality trait has been reported to be correlated with elevated parasympathetic cardiac control. $^{12}$

\section{Measurements of blood pressure, respiratory rate and heart rate variability}

Blood pressure (BP) was recorded with a Tensoval duo control Digital Blood Pressure Monitor (HARTMANN AG, Heidenheim, Germany). Systolic BP (SBP) and diastolic BP (DBP) were measured twice from the right arm during supine rest and then averaged. The detailed procedures for the analysis of HRV were as reported in our previous studies. ${ }^{23,31}$ Briefly, the subject first sat quietly for 20 minutes, then a lead I electrocardiogram was recorded for 5 minutes while they lay quietly in a supine position. To control diurnal changes in cardiac autonomic modulation, all electrocardiogram recordings were performed in a warm and quiet room between 9 and 11 a.m. Raw respiratory data during electrocardiogram recordings were obtained. The breathing rate was analysed and defined as the number of breaths per minute. An HRV analyzer (SSIC, Enjoy Research Inc., Taipei, Taiwan) acquired, stored, and processed the electrocardiography signals. Our computer algorithm then identified each QRS complex and rejected each ventricular premature complex or noise according to its likelihood in a standard QRS template. ${ }^{31}$ Signals were recorded at a sampling rate of $512 \mathrm{~Hz}$, using an 8-bit analogue-todigital converter. Stationary R-R interval values were re-sampled and interpolated at a rate of $7.11 \mathrm{~Hz}$ to produce continuity in the time domain. Power spectral analysis was performed using a non-parametric fast Fourier transformation. The direct current component was deleted, and a Hamming window was used to attenuate the leakage effect. ${ }^{23}$ The power spectrum was subsequently quantified into standard frequency-domain measurements, namely variance (variance of R-R-interval values), very low-frequency power (VLF, $0.003-0.04 \mathrm{~Hz}$ ), lowfrequency power (LF, $0.04-0.15 \mathrm{~Hz}$ ), high-frequency power (HF, $0.15-0.40 \mathrm{~Hz}$ ), and the ratio of LF to HF power (LF/HF). All of the measurements were logarithmically transformed to correct for a skewed distribution. ${ }^{32}$

Vagal control of HRV is represented by HF, whereas both vagal and sympathetic control of HRV are jointly represented by LF. The LF also reflects baroreflex function during supine rest. ${ }^{33}$ The LF/HF ratio could mirror sympatho-vagal balance or sympathetic modulation, with a larger LF/HF ratio indicating a greater predominance of sympathetic activity over cardiac vagal control. The VLF component has been attributed variously to thermoregulatory processes, peripheral vasomotor activity, and the renin-angiotensin system; however, its definite physiological meaning is under debate. ${ }^{34}$

\section{Statistical analyses}

SPSS version 19.0 (SPSS Inc., Chicago, IL, USA) statistical software was used for all analyses. Relationships between the $\mathrm{HRV}$ indices, control variables and the psychometric scores were analysed using Spearman correlation coefficients. Hierarchical regression analyses were used to explore the effect of personality trait on HRV profiles after adjusting for the psychological state and control variables. The psychological state and control variables relating to HRV in univariate analysis $(\mathrm{p}<0.05)$ were entered into step 1 of the hierarchical regression analysis, when mean R-R intervals and HRV indices were the dependent variables. Data including $\mathrm{R}^{2}, \mathrm{R}^{2}$-changes, $\mathrm{F}$, standardization regression coefficient $(\beta)$ and $p$ value in the regression model are presented. Tolerance and variance inflation factors were used to check for multicollinearity. In addition, participants were divided into two groups by HA scores. We took the highest quartile as the point of dichotomizing HA. The selection of the cut-off points in the present study was done through applying the categorization method using quartile values. ${ }^{35,36}$ Independent-samples t-tests were conducted to determine between-group differences in demographics and control variables. A multivariate analysis of variance (MANOVA) was performed to determine whether there were between-group differences on an interpretable composite of $\mathrm{HRV}$ variables across time and frequency-domain, and to provide a control for multiple comparisons. Analysis of covariance adjusted for any significant differences in baseline characteristics known to affect cardiac measures. A significant Pillai's trace effect for group was followed by univariate analyses of variance (ANOVAs) to identify which measures contributed to a significant multivariate effect. Findings were corrected for multiple comparisons via Bonferroni correction. Eta-squared ( $\eta 2$ ) was reported for ANOVA effects as an indicator of effect size (small $=0.01$, medium $=0.06$, large $=0.14$ ). The statistical analysis was conducted at a $95 \%$ confidence level. The $\alpha$-level was set at 0.05 per comparison.

\section{RESULTS}

\section{Sample characteristics}

The mean age of the subjects was $43.50 \pm 14.14$ years, the mean education level was $12.19 \pm 3.41$ years, the mean BMI was 
$23.09 \pm 3.45 \mathrm{~kg} / \mathrm{m}^{2}$, the mean SBP was $118.51 \pm 13.73 \mathrm{~mm} \mathrm{Hg}$, the mean DBP was $73.28 \pm 7.44 \mathrm{~mm} \mathrm{Hg}$, the mean exercise level was $1.30 \pm 2.23$ hours/week, the mean alcohol use was $0.01 \pm 0.02$ drinks/day and $54.4 \%$ were female. The scores from the BDI and BAI are presented in Table 1 . The results for the personality traits are presented in Table 1 also.

\section{Factors associated with HRV profiles}

The associations between control variables and resting HRV indices were shown in Table 2. Men had significantly higher LF and HF than women. Older participants had reduced variance (total HRV), VLF, LF and HF, and increased LF/HF ratio. Participants with higher BMI had lower HF but higher LF/HF ratio. Those who were habitually more physically active had significantly greater variance, LF and HF. Systolic/diastolic blood pressure showed no associations with RR interval and any HRV indices. With regard to the psychometric variables, the BAI and NS score showed no associations with RR interval or any HRV indices. The BDI score was associated with reduced RR interval and HF, and increased $\mathrm{LF} / \mathrm{HF}$ ratio. The HA score inversely correlated with the variance, $\mathrm{LF}$, and HF.

\section{Effects of harm avoidance on resting HRV profiles}

After adjusting for control variables and psychological state (BDI scores), the HA scores were still associated with reduced

Table 1. Sample characteristics

\begin{tabular}{|c|c|c|c|c|}
\hline Clinical and demographic data & $\mathrm{N}$ & $\%$ & Mean \pm SD & Range \\
\hline Males & 244 & 45.60 & & \\
\hline Females & 291 & 54.40 & & \\
\hline Age, years & 535 & & $43.50 \pm 14.14$ & $20.00-65.00$ \\
\hline Education, years & 535 & & $12.19 \pm 3.41$ & $6.00-22.00$ \\
\hline BMI, $\mathrm{kg} / \mathrm{m}^{2}$ & 535 & & $23.09 \pm 3.45$ & $16.82-34.17$ \\
\hline Weekly regular exercise, hours & 535 & & $1.30 \pm 2.23$ & $0.00-9.50$ \\
\hline Alcohol use, drinks/day & 535 & & $0.01 \pm 0.02$ & $0.00-0.15$ \\
\hline SBP, $\mathrm{mm} \mathrm{Hg}$ & 535 & & $118.51 \pm 13.73$ & $80.00-139.00$ \\
\hline DBP, $\mathrm{mm} \mathrm{Hg}$ & 535 & & $73.28 \pm 7.44$ & $55.00-89.00$ \\
\hline BDI scores & 535 & & $5.58 \pm 3.77$ & $0.00-15.00$ \\
\hline BAI scores & 535 & & $8.11 \pm 2.69$ & $1.00-13.00$ \\
\hline NS scores & 535 & & $14.94 \pm 4.14$ & $5.00-30.00$ \\
\hline HA scores & 535 & & $18.16 \pm 7.59$ & $4.00-30.00$ \\
\hline
\end{tabular}

SD: standard deviation, SBP: systolic blood pressure, DBP: diastolic blood pressure, BMI: body mass index (calculated as weight in kilograms divided by height in meters squared), BDI: Beck Depression Inventory, BAI: Beck Anxiety Inventory, NS: novelty seeking, HA: harm avoidance

Table 2. Factors associated with resting HRV indices among all participants

\begin{tabular}{lcccccc}
\hline & Mean RR intervals & Var & VLF & LF & HF & LF/HF \\
\hline Sex (women/men) ${ }^{\dagger}$ & 0.05 & 0.08 & 0.06 & $0.15^{* * *}$ & $0.09^{*}$ & 0.04 \\
Age $^{\ddagger}$ & 0.05 & $-0.41^{* * *}$ & $-0.33^{* * *}$ & $-0.45^{* * *}$ & $-0.45^{* * *}$ & $0.13^{* *}$ \\
BMI $^{\ddagger}$ & -0.07 & -0.07 & -0.05 & -0.06 & $-0.13^{* *}$ & $0.14^{* *}$ \\
Physical activity $^{\ddagger}$ & 0.01 & $0.09^{*}$ & 0.06 & $0.10^{*}$ & $0.10^{*}$ & -0.02 \\
Alcohol use $^{\ddagger}$ & 0.04 & -0.04 & -0.06 & -0.07 & -0.03 & -0.03 \\
SBP $^{\ddagger}$ & -0.06 & -0.07 & -0.09 & -0.07 & -0.07 & 0.02 \\
DBP $^{\ddagger}$ & -0.03 & 0.01 & 0.02 & 0.01 & 0.04 & -0.08 \\
BDI scores $^{\ddagger}$ & $-1.1^{*}$ & -0.05 & -0.06 & -0.04 & $-0.11^{* *}$ & $0.16^{* * *}$ \\
BAI scores $^{\ddagger}$ & 0.02 & -0.03 & 0.01 & -0.05 & -0.01 & -0.03 \\
NS scores $^{\ddagger}$ & 0.06 & 0.03 & 0.04 & 0.05 & -0.05 & -0.06 \\
HA scores $^{\ddagger}$ & -0.07 & $-0.10^{*}$ & -0.05 & $-0.10^{*}$ & $-0.12^{* *}$ & 0.02 \\
\hline
\end{tabular}

${ }^{*} \mathrm{p}<0.05,{ }^{* *} \mathrm{p}<0.01,{ }^{* * *} \mathrm{p}<0.001,{ }^{\dagger}$ point-biserial correlations; first category in parenthesis is the reference group, ${ }^{\ddagger}$ product-moment correlations. BMI: body mass index, SBP: systolic blood pressure, DBP: diastolic blood pressure, Var: total variance $\left[\ln \left(\mathrm{ms}^{2}\right)\right]$, VLF: very low-frequency power $\left[\ln \left(\mathrm{ms}^{2}\right)\right]$, LF: low frequency power $\left[\ln \left(\mathrm{ms}^{2}\right)\right]$, HF: high frequency power $\left[\ln \left(\mathrm{ms}^{2}\right)\right]$, LF/HF: ratio of LF to HF [ln(ratio)], BDI: Beck Depression Inventory, BAI: Beck Anxiety Inventory, NS: novelty seeking, HA: harm avoidance 
variance, LF and HF (Table 3). Tolerance (range: 0.952-0.999) and variance inflation (range: 1.001-1.150) did not indicate multicollinearity.

\section{Heart rate variability parameters: high HA group versus low HA group}

The highest quartile value of the HA scores of the 535 participants was 25.0. The mean breathing rate was $13.48 \pm 3.40$ breaths/min for the subjects with HA scores above the upper quartile (high HA group) and 13.78 \pm 3.23 breaths/min for those with HA scores below the upper quartile (low HA group). The breathing rate was comparable for the two groups $(t=0.99, p=0.33)$. The high HA group had lower BDI scores but higher BAI scores as compared to the low HA group (Table 4). The MANOVA comparing the various HRV measures between high HA and low HA groups showed significant results $[F(6,526)=2.81, p=0.01, \eta 2=0.031]$ (Figure 1). The result did not significantly alter after adjusting for BDI and BAI scores $[F(6,526)=3.36, p=0.003, \eta 2=0.037]$. A separate ANOVA was conducted for each dependent variable, with each ANOVA evaluated at an alpha level of $0.0083(0.05 / 6)$. Compared with the low HA group, high HA group displayed significant reductions in variance $[\mathrm{F}(1,531)=7.37, \mathrm{p}=0.007, \eta 2=0.014], \mathrm{LF}[\mathrm{F}$ $(1,531)=7.71, \mathrm{p}=0.006, \eta 2=0.014]$ and $\operatorname{HF}[\mathrm{F}(1,531)=17.09$, $\mathrm{p}<0.001, \eta 2=0.031]$ and a significant increase in their LF/HF ratio $[F(1,531)=7.89, \mathrm{p}=0.005, \eta 2=0.015]$. However, the groups did not significantly differ on mean R-R intervals $[F$ $(1,531)=6.65, \mathrm{p}=0.01, \eta 2=0.012]$ or $\operatorname{VLF}[\mathrm{F}(1,531)=4.55, \mathrm{p}=$ $0.033, \eta 2=0.008]$.

\section{DISCUSSION}

The present study focuses on the relationship between autonomic functioning and harm avoidance, an anxiety-related personality trait, in a large and well-defined healthy population. Our results support the hypothesis that meaningful correlation exists between autonomic functioning and harm avoidance and suggest that a decrease in HRV may represent

Table 3. Hierarchical regression analyses of mean RR intervals and all HRV indices

\begin{tabular}{|c|c|c|c|c|c|c|c|c|c|c|c|c|}
\hline & \multicolumn{12}{|c|}{ Standardized regression coefficient and p-value } \\
\hline & \multicolumn{2}{|c|}{ Mean RR intervals } & \multicolumn{2}{|c|}{ Var } & \multicolumn{2}{|c|}{ VLF } & \multicolumn{2}{|c|}{ LF } & \multicolumn{2}{|c|}{$\mathrm{HF}$} & \multicolumn{2}{|c|}{ Ratio } \\
\hline & $\beta$ & $\mathrm{p}$ & $\beta$ & $\mathrm{p}$ & $\beta$ & $\mathrm{p}$ & $\beta$ & $\mathrm{p}$ & $\beta$ & $\mathrm{p}$ & $\beta$ & $\mathrm{p}$ \\
\hline \multicolumn{13}{|l|}{ Univariate analyses } \\
\hline Gender & 0.05 & 0.22 & 0.08 & 0.06 & 0.06 & 0.14 & 0.15 & $<0.001$ & 0.09 & 0.046 & 0.04 & 0.36 \\
\hline Age & 0.05 & 0.30 & -0.41 & $<0.001$ & -0.33 & $<0.001$ & -0.45 & $<0.001$ & -0.45 & $<0.001$ & 0.13 & 0.002 \\
\hline $\mathrm{BMI}$ & -0.07 & 0.11 & -0.07 & 0.10 & -0.05 & 0.22 & -0.06 & 0.20 & -0.13 & 0.002 & 0.14 & 0.002 \\
\hline Physical activity & 0.01 & 0.86 & 0.09 & 0.04 & 0.06 & 0.18 & 0.10 & 0.03 & 0.10 & 0.02 & -0.02 & 0.62 \\
\hline BDI & -0.11 & 0.01 & -0.05 & 0.25 & -0.06 & 0.15 & -0.04 & 0.38 & -0.11 & 0.013 & 0.16 & $<0.001$ \\
\hline \multicolumn{13}{|l|}{ Step 1} \\
\hline Gender & & & & & & & 0.08 & 0.046 & 0.04 & 0.33 & & \\
\hline Age & & & -0.41 & $<0.001$ & -0.33 & $<0.001$ & -0.44 & $<0.001$ & -0.47 & $<0.001$ & 0.15 & $<0.001$ \\
\hline BMI & & & & & & & & & -0.11 & 0.004 & 0.13 & 0.002 \\
\hline Physical activity & & & 0.10 & 0.01 & & & 0.11 & 0.005 & 0.10 & 0.009 & & \\
\hline $\mathrm{BDI}$ & -0.11 & 0.01 & & & & & & & -0.19 & $<0.001$ & 0.19 & $<0.001$ \\
\hline $\mathrm{R}^{2}$ & \multicolumn{2}{|c|}{$1.1 \%$} & \multicolumn{2}{|c|}{$17.5 \%$} & \multicolumn{2}{|c|}{$10.9 \%$} & \multicolumn{2}{|c|}{$22.3 \%$} & \multicolumn{2}{|c|}{$26.2 \%$} & \multicolumn{2}{|c|}{$6.7 \%$} \\
\hline \multicolumn{13}{|l|}{ Step 2} \\
\hline Gender & & & & & & & 0.08 & 0.04 & 0.04 & 0.31 & & \\
\hline Age & & & -0.41 & $<0.001$ & -0.33 & $<0.001$ & -0.44 & $<0.001$ & -0.47 & $<0.001$ & 0.15 & $<0.001$ \\
\hline BMI & & & & & & & & & -0.11 & 0.005 & 0.13 & 0.002 \\
\hline Physical activity & & & 0.10 & 0.01 & & & 0.11 & 0.004 & 0.10 & 0.006 & & \\
\hline BDI & -0.11 & 0.01 & & & & & & & -0.20 & $<0.001$ & 0.19 & $<0.001$ \\
\hline $\mathrm{HA}$ & -0.09 & 0.05 & -0.09 & 0.016 & -0.04 & 0.34 & -0.09 & 0.014 & -0.13 & 0.001 & 0.03 & 0.47 \\
\hline $\mathrm{R}^{2}$-changes & \multicolumn{2}{|c|}{$0.7 \%$} & \multicolumn{2}{|c|}{$0.9 \%$} & \multicolumn{2}{|c|}{$0.2 \%$} & \multicolumn{2}{|c|}{$0.9 \%$} & \multicolumn{2}{|c|}{$1.6 \%$} & \multicolumn{2}{|c|}{$0.1 \%$} \\
\hline $\mathrm{F}$ & \multicolumn{2}{|c|}{3.96} & \multicolumn{2}{|c|}{5.79} & \multicolumn{2}{|c|}{0.93} & \multicolumn{2}{|c|}{6.3} & \multicolumn{2}{|c|}{11.90} & \multicolumn{2}{|c|}{1.55} \\
\hline $\mathrm{p}$ value & \multicolumn{2}{|c|}{$\mathrm{p}=0.05$} & \multicolumn{2}{|c|}{$\mathrm{p}=0.016$} & & .34 & & .014 & & .001 & & \\
\hline
\end{tabular}

BMI: body mass index, BDI: Beck Depression Inventory, HA: harm avoidance 
Table 4. Characteristic differences between participants with high harm avoidance and low harm avoidance

\begin{tabular}{lccc}
\hline \multicolumn{1}{c}{ Clinical and demographic data } & $\begin{array}{c}\text { High Harm Avoidance } \\
\text { HA scores } \geq 25(\mathrm{~N}=171)\end{array}$ & $\begin{array}{c}\text { Low Harm Avoidance } \\
\text { HA scores }<25(\mathrm{~N}=364)\end{array}$ & $\begin{array}{c}\text { Omnibus } \\
\text { p-value }\end{array}$ \\
\hline Female sex $(\%)$ & $93(54.40)$ & $198(54.40)$ & 1.00 \\
Education levels, mean \pm SD, years & $11.97 \pm 3.50$ & $12.30 \pm 3.37$ & 0.30 \\
Age, mean \pm SD, years & $44.77 \pm 13.75$ & $42.91 \pm 14.31$ & 0.16 \\
BMI, mean \pm SD, kg/m ${ }^{2}$ & $23.39 \pm 3.61$ & $22.94 \pm 3.62$ & 0.16 \\
Weekly regular exercise, hours & $1.38 \pm 2.38$ & $1.23 \pm 2.13$ & 0.55 \\
Alcohol use, drinks/day & $0.005 \pm 0.02$ & $0.006 \pm 0.02$ & 0.71 \\
SBP, mm Hg & $119.44 \pm 13.91$ & $118.07 \pm 13.65$ & 0.28 \\
DBP, mm Hg & $73.04 \pm 7.62$ & $73.39 \pm 7.36$ & 0.61 \\
BDI scores, mean \pm SD & $5.05 \pm 3.86$ & $5.82 \pm 3.70$ & 0.03 \\
BAI scores, mean \pm SD & $8.84 \pm 2.73$ & $7.77 \pm 2.61$ & $<0.001$
\end{tabular}

SD: standard deviation, BMI: body mass index (calculated as weight in kilograms divided by height in meters squared), SBP: systolic blood pressure, DBP: diastolic blood pressure, BDI: Beck Depression Inventory, BAI: Beck Anxiety Inventory

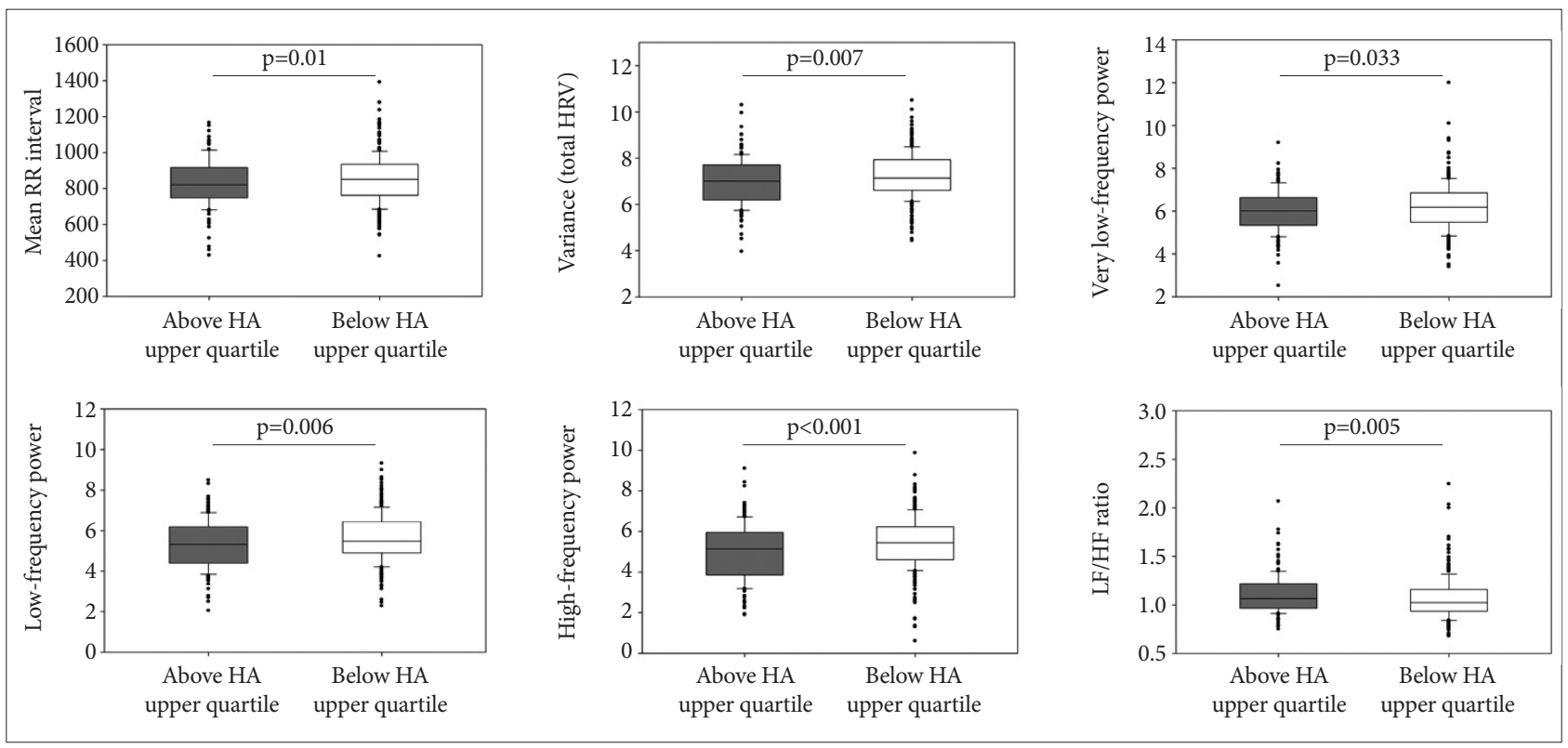

Figure 1. Comparison of mean R-R intervals and all measures of HRV between participants with high and low harm avoidance. Boxes indicate data between the 25 th and 75 th percentile with the horizontal bar reflecting the median.

a psychophysiological biomarker associated with anxiety personality traits in the general population. The main results of our study may be summed as follows.

Our present results that controlled for potential confounding factors (i.e., psychological state and other control variables) showed a significant contribution of HA temperament to reduced HRV and vagal modulation of heart rate. In this regard, our results confirm findings by prior studies. ${ }^{4,37,38}$ Consistent with these associations, when our participants were divided into two groups by HA scores, those with high HA exhibited significantly lower HRV and vagal activity as compared with those with low HA. The significant differences were not due to the levels of subclinical depression and/or anxiety because adjusting for psychological states did not alter the significance. Moreover, it should be stressed that the difference in mean BAI scores between high and low HA groups may be statistically significant due to a large sample size, but not be clinically significant because mean BAI scores of both groups are only within the borderline of minimal to mild level of anxiety (score 7-8). The same applied to mean BDI scores.

In addition, we here demonstrated low LF power in participants with high HA. In the present study the LF power when assessed in the supine position is primarily a result of baroreflex sensitivity, which is a measure of the gain of the baroreflex. ${ }^{33,39}$ Thus, high HA may be associated with lower baroreflex functioning, while reduced baroreflex sensitivity per se has been demonstrated to be a valuable and independent predictor of sudden cardiac death after myocardial infarction. ${ }^{40}$ 
There could be two possible explanations for all these findings. First, HA corresponds to an inhibitory response to signals of aversive stimuli that leads to avoidance of punishment and is theoretically associated with serotonergic activity. ${ }^{10,41}$ For example, findings reveal that the HA score is positively associated with serotonin (5-HT) receptor sensitivity. ${ }^{41}$ Based on previous findings, a high HA score seems to reflect lower 5-HT function. Next, an association between lower 5-HT function and reduced HRV is established by recent evidence from a rodent study indicating that 5-HT depletion or 5-HT receptor blocking attenuates baroreflex gain. ${ }^{42}$ The arterial baroreflex is the main mediator of HRV. ${ }^{43}$ Therefore, lowered 5-HT function leads to diminished baroreflex function, which in turn leads to reduced HRV. This explanation is compatible with the negative correlation between HA score and LF power observed in our study.

Second, previous evidence indicated that 5-HT has a direct central effect on $\mathrm{HRV}^{44}$ and cardiac vagal control. ${ }^{45}$ This could be another reason that HA is linked to vagal modulation of the heart rate. Collectively, our study demonstrates that healthy individuals with high HA tend to have decreased HRV, vagal activity and possible baroreflex function, providing a potential underlying mechanism for stable trait anxiety to confer increased risk for CVD. ${ }^{7-9}$ A previous study, however, reported that a low level of HA is related to a high level of physiologic CVD risk factors in young men. ${ }^{46}$ Future longitudinal studies are warranted to establish whether HA predisposes an individual to the development of CVD in a sex-specific manner.

We found that normative variation in self-reported depression state rather than anxiety state was associated with reduced HRV, which corroborate findings of the largest study published so far. ${ }^{5}$ For physically and mentally healthy adults, it is highly possible that anxiety trait instead of non-clinical anxiety state could explain the variations in HRV. In fact, there is a high degree of overlap and strong linkage between HA and generalized anxiety disorder (GAD), a disorder characterized by worry and hypervigilance; for example, both of them share features of feeling habitually tense and worried. In addition, previous evidence indicated that HA scores were highly predictive of later GAD diagnoses ${ }^{47}$ and that depressed patients with comorbid GAD compared to those without comorbidity were associated with higher HA. ${ }^{48}$ Recent HRV studies in clinical samples have focused on GAD. The largest case-control study published so far reported that among a variety of mood and anxiety disorders, only the diagnosis of GAD was associated with robust decreases in $\mathrm{HRV}^{49}$ Moreover, among depressed patients with and without comorbid anxiety disorders, those with comorbid GAD had the greatest reduction in $\mathrm{HRV} .^{50}$ A plausible scenario may be that high HA reflect a physiological system that is less flexible, less able to appropri- ately respond to environment demands, and, thus, more susceptible to ensuing disease processes especially associated with autonomic imbalance (e.g., GAD). Such a possibility would prompt the further investigation into whether longterm reduction in HRV may precede the development of clinically significant anxiety symptoms for healthy individuals with high HA being later diagnosed with GAD.

Our regression analysis failed to show a significant correlation between HA and LF/HF ratio, although participants with high HA exhibited increased LF/HF ratio as compared to those with low HA. In the present study, LF power is more likely to represent baroreflex function rather than sympathetic activity. In such a situation, the LF/HF ratio only poorly reflects sympathovagal balance, and is more accurately a measure of the relationship between vagal input and other sources of variability. ${ }^{51}$ As we did not use a pure measure of sympathetic activity in this study, we cannot say whether sympathetic tone was increased. Additional studies using cardiac noradrenaline spillover, the pre-ejection period, or muscle sympathetic nerve activity, are needed to adequately assess sympathetic tone in these subjects.

Contrary to our data, an earlier report described that NS correlated with higher parasympathetic activity but the association attenuated to the null after adjustments for health-related behaviours. ${ }^{12}$ The authors explained that novelty seekers may have naturally high levels of parasympathetic activity but are also likely to be stimulant users; the co-existence of these factors renders the association non-significant. Nonetheless, our results are comparatively easier to interpret since the present study used the sample free of adverse health-related behaviours. To be conclusive, future studies should investigate the association between NS and HRV longitudinally.

We believe that our study has a number of strengths that reinforce the reliability of our results. First, depression and anxiety have been related to HRV among cardiac ${ }^{52}$ and psychiatric patients, ${ }^{2,3}$ but not consistently among healthy or otherwise unselected samples. ${ }^{4-6,37}$ It is conceivable that any associations observed in healthy samples may be driven by a subset of individuals who have not been evaluated formally but nonetheless meet the criteria for mood or anxiety disorder. All participants in the present study were evaluated with a structured diagnostic interview. This type of screening ruled out current or past psychiatric disorders. Second, none of the study subjects were current or past smokers. Smoking clearly depresses HRV, even among those who have recently quit, and HRV remains lower compared to that of normal non-smokers. ${ }^{53}$ Finally, all participants underwent relevant laboratory investigations in addition to self-reported data of physical health. Our recent studies have emphasized this objective procedure for excluding subjects with physical co-morbidities, 
since subjects might underestimate their biological risk factors (e.g., elevated glucose and atherogenic lipid profile) for cardiac autonomic dysregulation when these factors were self-reported. ${ }^{54,55}$ Collectively, our sample was well suited for studying HRV, as the effects of potential confounding factors were minimized.

The present study has two limitations. First, the presented regression analyses in our study provide several significant associations but the level of correlation (or regression coefficient) is low and insofar may be not physiologically relevant. The results are providing exploratory information about possible relations which should be investigated in further research. Second, we classified the healthy individuals into high or low HA groups according to only the quartiles of the sample HA scores. The optimum HA scores to dichotomize patients and to produce the largest contribution of a prognostic predictor to the physiologically relevant reduction in HRV should be explored in future research.

\section{Conclusions}

This study highlights the importance of harm avoidance in independently contributing to decreased cardiac autonomic regulation in healthy adults and provides a new insight of the linkage between anxiety trait and increased risk of cardiovascular disease.

\section{Acknowledgments}

This study was supported in part by grants from the Ministry of Science and Technology of the Taiwanese Government (MOST-103-2314-B-016-021), the Tri-Service General Hospital (TSGH-C103-135), and the National Defense Medical Research (MAB-104-008).

\section{REFERENCES}

1. Taylor CB. Depression, heart rate related variables and cardiovascular disease. Int J Psychophysiol 2010;78:80-88.

2. Cohen H, Benjamin J. Power spectrum analysis and cardiovascular morbidity in anxiety disorders. Auton Neurosci 2006;128:1-8.

3. Kemp AH, Quintana DS, Gray MA, Felmingham KL, Brown K, Gatt JM. Impact of depression and antidepressant treatment on heart rate variability: a review and meta-analysis. Biol Psychiatry 2010;67:10671074.

4. Bleil ME, Gianaros PJ, Jennings JR, Flory JD, Manuck SB. Trait negative affect: toward an integrated model of understanding psychological risk for impairment in cardiac autonomic function. Psychosom Med 2008; 70:328-337.

5. Ohira T, Diez Roux AV, Prineas RJ, Kizilbash MA, Carnethon MR, Folsom AR. Associations of psychosocial factors with heart rate and its short-term variability: multi-ethnic study of atherosclerosis. Psychosom Med 2008;70:141-146.

6. Virtanen R, Jula A, Salminen JK, Voipio-Pulkki LM, Helenius H, Kuusela $\mathrm{T}$, et al. Anxiety and hostility are associated with reduced baroreflex sensitivity and increased beat-to-beat blood pressure variability. Psychosom Med 2003;65:751-756.

7. Paterniti S, Zureik M, Ducimetiere P, Touboul PJ, Feve JM, Alperovitch A. Sustained anxiety and 4-year progression of carotid atherosclerosis. Arterioscler Thromb Vasc Biol 2001;21:136-141.
8. Hernandez R, Allen NB, Liu K, Stamler J, Reid KJ, Zee PC, et al. Association of depressive symptoms, trait anxiety, and perceived stress with subclinical atherosclerosis: results from the Chicago Healthy Aging Study (CHAS). Prev Med 2014;61:54-60.

9. Sovio U, King V, Miettunen J, Ek E, Laitinen J, Joukamaa M, et al. Cloninger's Temperament dimensions, socio-economic and lifestyle factors and metabolic syndrome markers at age 31 years in the Northern Finland Birth Cohort 1966. J Health Psychol 2007;12:371-382.

10. Cloninger CR. Neurogenetic adaptive mechanisms in alcoholism. Science 1987;236:410-416.

11. Battaglia M, Przybeck TR, Bellodi L, Cloninger CR. Temperament dimensions explain the comorbidity of psychiatric disorders. Compr Psychiatry 1996;37:292-298.

12. Puttonen S, Elovainio M, Kivimaki M, Koskinen T, Pulkki-Råback L, Viikari JS, et al. Temperament, health-related behaviors, and autonomic cardiac regulation: the cardiovascular risk in young Finns study. Biol Psychol 2008;78:204-210.

13. Huang WL, Chang LR, Kuo TB, Lin YH, Chen YZ, Yang CC. Gender differences in personality and heart-rate variability. Psychiatry Res 2013; 209:652-657.

14. Thayer JF, Hansen AL, Saus-Rose E, Johnsen BH. Heart rate variability, prefrontal neural function, and cognitive performance: the neurovisceral integration perspective on self-regulation, adaptation, and health. Ann Behav Med 2009;37:141-153.

15. Thayer JF, Lane RD. A model of neurovisceral integration in emotion regulation and dysregulation. J Affect Disord 2000;61:201-216.

16. Dekker JM, Crow RS, Folsom AR, Hannan PJ, Liao D, Swenne CA, et al. Low heart rate variability in a 2-minute rhythm strip predicts risk of coronary heart disease and mortality from several causes: the ARIC Study. Atherosclerosis Risk In Communities. Circulation 2000;102: 1239-1244.

17. Heart rate variability: standards of measurement, physiological interpretation and clinical use. Task Force of the European Society of Cardiology and the North American Society of Pacing and Electrophysiology. Circulation 1996;93:1043-1065.

18. Chang HA, Chang CC, Kuo TB, Huang SY. Distinguishing bipolar II depression from unipolar major depressive disorder: differences in heart rate variability. World J Biol Psychiatry 2015;16:351-360.

19. Chang HA, Chang CC, Tzeng NS, Kuo TB, Lu RB, Huang SY. Generalized anxiety disorder, comorbid major depression and heart rate variability: a case-control study in taiwan. Psychiatry Investig 2013;10:326335.

20. Yeh TC, Kao LC, Tzeng NS, Kuo TB, Huang SY, Chang CC, et al. Heart rate variability in major depressive disorder and after antidepressant treatment with agomelatine and paroxetine: findings from the Taiwan Study of Depression and Anxiety (TAISDA). Prog Neuropsychopharmacol Biol Psychiatry 2016;64:60-67.

21. Endicott J, Spitzer RL. A diagnostic interview: the schedule for affective disorders and schizophrenia. Arch Gen Psychiatry 1978;35:837-844.

22. Chen TY, Chang CC, Tzeng NS, Terry Kuo BJ, Huang SY, Lu RB, et al. Different patterns of heart rate variability during acute withdrawal in alcohol dependent patients with and without comorbid anxiety and/or depression. J Psychophysiol 2015;29:87-98.

23. Kuo TB, Lin T, Yang CC, Li CL, Chen CF, Chou P. Effect of aging on gender differences in neural control of heart rate. Am J Physiol 1999; 277:H2233-H2239.

24. Antelmi I, de Paula RS, Shinzato AR, Peres CA, Mansur AJ, Grupi CJ. Influence of age, gender, body mass index, and functional capacity on heart rate variability in a cohort of subjects without heart disease. Am J Cardiol 2004;93:381-385.

25. Wu JS, Lu FH, Yang YC, Lin TS, Huang YH, Wu CH, et al. Epidemiological evidence of altered cardiac autonomic function in overweight but not underweight subjects. Int J Obes (Lond) 2008;32:788-794.

26. Rosenwinkel ET, Bloomfield DM, Arwady MA, Goldsmith RL. Exercise and autonomic function in health and cardiovascular disease. Cardiol 
Clin 2001;19:369-387.

27. Henje Blom E, Olsson EM, Serlachius E, Ericson M, Ingvar M. Heart rate variability is related to self-reported physical activity in a healthy adolescent population. Eur J Appl Physiol 2009;106:877-883.

28. Babor TF, Fuente JR, de la Saunders J, Grant M. The Alcohol Use Disorders Identification Test: Guidelines for Use in Primary Health Care. Geneva, Switzerland: World Health Organization; 1992.

29. Li Q, Babor TF, Hao W, Chen X. The Chinese translations of Alcohol Use Disorders Identification Test (AUDIT) in China: a systematic review. Alcohol Alcohol 2011;46:416-423.

30. Chen WJ, Chen HM, Chen CC, Yu WY, Cheng AT. Cloninger's Tridimensional Personality Questionnaire: psychometric properties and construct validity in Taiwanese adults. Compr Psychiatry 2002;43:158166.

31. Liu CC, Kuo TB, Yang CC. Effects of estrogen on gender-related autonomic differences in humans. Am J Physiol Heart Circ Physiol 2003; 285:H2188-H2193.

32. Ellis RJ, Sollers Iii JJ, Edelstein EA, Thayer JF. Data transforms for spectral analyses of heart rate variability. Biomed Sci Instrum 2008;44:392-397.

33. Goldstein DS, Bentho O, Park MY, Sharabi Y. Low-frequency power of heart rate variability is not a measure of cardiac sympathetic tone but may be a measure of modulation of cardiac autonomic outflows by baroreflexes. Exp Physiol 2011;96:1255-1261.

34. Lombardi F. Clinical implications of present physiological understanding of HRV components. Card Electrophysiol Rev 2002;6:245-249.

35. Farmer RF, Seeley JR. Temperament and character predictors of depressed mood over a 4-year interval. Depress Anxiety 2009;26:371-381.

36. Asano T, Baba H, Kawano R, Takei H, Maeshima H, Takahashi Y, et al. Temperament and character as predictors of recurrence in remitted patients with major depression: a 4-year prospective follow-up study. Psychiatry Res 2015;225:322-325.

37. Dishman RK, Nakamura Y, Garcia ME, Thompson RW, Dunn AL, Blair $\mathrm{SN}$. Heart rate variability, trait anxiety, and perceived stress among physically fit men and women. Int J Psychophysiol 2000;37:121-133.

38. Ode S, Hilmert CJ, Zielke DJ, Robinson MD. Neuroticism's importance in understanding the daily life correlates of heart rate variability. Emotion 2010;10:536-543.

39. Moak JP, Goldstein DS, Eldadah BA, Saleem A, Holmes C, Pechnik S, et al. Supine low-frequency power of heart rate variability reflects baroreflex function, not cardiac sympathetic innervation. Heart Rhythm 2007; 4:1523-1529.

40. La Rovere MT, Bigger JT Jr, Marcus FI, Mortara A, Schwartz PJ. Baroreflex sensitivity and heart-rate variability in prediction of total cardiac mortality after myocardial infarction. ATRAMI (Autonomic Tone and Reflexes After Myocardial Infarction) Investigators. Lancet 1998;351: 478-484.

41. Peirson AR, Heuchert JW, Thomala L, Berk M, Plein H, Cloninger CR.
Relationship between serotonin and the temperament and character inventory. Psychiatry Res 1999;89:29-37.

42. Damaso EL, Bonagamba LG, Kellett DO, Jordan D, Ramage AG, Machado BH. Involvement of central 5-HT7 receptors in modulation of cardiovascular reflexes in awake rats. Brain Res 2007;1144:82-90.

43. Frederiks J, Swenne CA, TenVoorde BJ, Honzíková N, Levert JV, Maan $\mathrm{AC}$, et al. The importance of high-frequency paced breathing in spectral baroreflex sensitivity assessment. J Hypertens 2000;18:1635-1644.

44. Shields RW Jr. Functional anatomy of the autonomic nervous system. J Clin Neurophysiol 1993;10:2-13.

45. Jordan D. Vagal control of the heart: central serotonergic (5-HT) mechanisms. Exp Physiol 2005;90:175-181.

46. Keltikangas-Jarvinen L, Ravaja N, Viikari J. Identifying Cloninger's temperament profiles as related to the early development of the metabolic cardiovascular syndrome in young men. Arterioscler Thromb Vasc Biol 1999;19:1998-2006.

47. Rettew DC, Doyle AC, Kwan M, Stanger C, Hudziak JJ. Exploring the boundary between temperament and generalized anxiety disorder: a receiver operating characteristic analysis. J Anxiety Disord 2006;20: 931-945.

48. Goldberg DP, Wittchen HU, Zimmermann P, Pfister H, Beesdo-Baum K. Anxious and non-anxious forms of major depression: familial, personality and symptom characteristics. Psychol Med 2014;44:1223-1234.

49. Kemp AH, Brunoni AR, Santos IS, Nunes MA, Dantas EM, Carvalho de Figueiredo R, et al. Effects of depression, anxiety, comorbidity, and antidepressants on resting-state heart rate and its variability: an ELSABrasil cohort baseline study. Am J Psychiatry 2014;171:1328-1334.

50. Kemp AH, Quintana DS, Felmingham KL, Matthews S, Jelinek HF. Depression, comorbid anxiety disorders, and heart rate variability in physically healthy, unmedicated patients: implications for cardiovascular risk. PLoS One 2012;7:e30777.

51. Malliani A. Heart rate variability: from bench to bedside. Eur J Intern Med 2005;16:12-20.

52. Francis JL, Weinstein AA, Krantz DS, Haigney MC, Stein PK, Stone $\mathrm{PH}$, et al. Association between symptoms of depression and anxiety with heart rate variability in patients with implantable cardioverter defibrillators. Psychosom Med 2009;71:821-827.

53. Stein PK, Rottman JN, Kleiger RE. Effect of $21 \mathrm{mg}$ transdermal nicotine patches and smoking cessation on heart rate variability. Am J Cardiol 1996;77:701-705.

54. Chang CC, Fang WH, Chang HA, Chen TY, Huang SY. Sex-specific association between nerve growth factor polymorphism and cardiac vagal modulation. Psychosom Med 2014;76:638-643.

55. Chang CC, Chang HA, Chen TY, Fang WH, Huang SY. Brain-derived neurotrophic factor (BDNF) Val66Met polymorphism affects sympathetic tone in a gender-specific way. Psychoneuroendocrinology 2014; 47:17-25. 